\title{
Association of plasma osteoprotegerin levels with the severity of lower extremity arterial disease in patients with type 2 diabetes
}

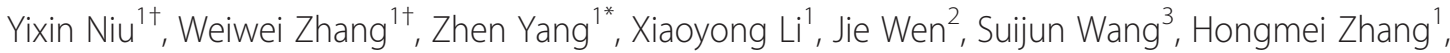
Xuanchun Wang ${ }^{2}$, Houguang Zhou ${ }^{4}$, Wenjun Fang ${ }^{1}$, Li Qin ${ }^{1}$ and Qing Su ${ }^{1 *}$

\begin{abstract}
Background: Osteoprotegerin (OPG) is a member of the tumor necrosis factor receptor superfamily and suggested as a marker of atherosclerosis. However, little is known about the association between plasma OPG levels and lower extremity arterial disease. We investigated whether plasma OPG levels were associated with the presence and severity of lower extremity arterial disease in patients with type 2 diabetes.

Methods: This was a study of 712 patients with type 2 diabetes aged 40 years or older. Plasma OPG was measured using ELISA. The lower extremity arterial disease was diagnosed by high-frequency color Doppler ultrasonic.

Results: Of 712 patients, 505 (70.9\%) had lower extremity arterial stenosis. OPG levels were significantly increased in patients with lower extremity arterial stenosis [1.89 (1.48-2.41) vs. $2.39(1.82-3.33) \mathrm{ng} / \mathrm{mL}, p<0.001]$. Plasma OPG levels increased gradually with increasing severity of lower extremity arterial stenosis ( $p<0.001$ for trend), after adjustment for traditional cardiovascular risk factors such as age, gender, smoking, total cholesterol, high-density lipoprotein (HDL) cholesterol, C-reactive protein (CRP), body mass index (BMI), systolic blood pressure(SBP). The risk of lower extremity arterial disease was increased $(\mathrm{OR}=1.17,95 \% \mathrm{Cl} 1.09-1.28, p<0.001)$ with each standard deviation (SD) higher level of OPG in patients with type 2 diabetes after adjustment for traditional CVD risk factors.

Conclusions: Plasma OPG levels were significantly associated with the presence and severity of lower extremity arterial disease. Our results suggest that OPG is an important plasma biomarker of lower extremity arterial disease in type 2 diabetes.
\end{abstract}

\section{Backgrounds}

Osteoprotegerin (OPG) is a soluble member of the TNF-receptor superfamily and act as decoy receptor for both the receptor activator of nuclear factor-B ligand (RANK-L) and the TNF-related apoptosis inducing ligand (TRAIL), two cytokines of the TNFfamily [1]. OPG is produced by a variety of tissues, including the cardiovascular system, lung, kidney, and immune tissues, as well as bone, and that the expression and production of OPG are regulated by various cytokines and hormones [2].

\footnotetext{
*Correspondence: yangzhen1020@hotmail.com; suqingxinhua@163.com ${ }^{\dagger}$ Equal contributors

'Department of Endocrinology, Xinhua Hospital Affiliated to Shanghai Jiaotong University School of Medicine, 1665 Kongjiang Road, Shanghai, China

Full list of author information is available at the end of the article
}

Recent studies have indicated that OPG also acts as an important regulatory molecule in vascular disease, such as arterial calcification and atherosclerosis [3-5]. The function of OPG in the arterial wall is not known, but it has been suggested that the molecule acts as a vascular calcification inhibitor due to the fact that OPG knock-out mice develop arterial calcifications [6]. Atherosclerosis is now understood to be an inflammatory disease, and not merely the result of the passive accumulation of lipids within arterial walls [7]. OPG is expressed in vascular cells such as coronary smooth muscle cells and endothelial cells in vitro. In endothelial cells, OPG has been demonstrated to act as an anti-apoptotic factor. Moreover, OPG immunoreactivity was demonstrated not only in the nondiseased vessel wall, but also in early atherosclerotic lesions in human tissues. These findings suggested that OPG may play a pivotal role in the development of vascular 
disease. A clinical study reported that there was a significant correlation between elevated serum OPG levels and cardiovascular mortality, suggesting that OPG may contribute to the progression of coronary artery disease (CAD), and that they were increased in elderly people and in diabetic patients [8].

Several studies have suggested that circulating OPG levels is positively associated with peripheral arterial disease $[9,10]$, which defined using ankle brachial index $(\mathrm{ABI})$, although data is inconsistent in recent meta analysis [11]. However, little is known about the association between plasma OPG levels and lower extremity arterial disease, which diagnosed by ultrasonic evaluation, in patients with type 2 diabetes. In this study, we investigated whether plasma OPG levels were associated with the presence and severity of lower extremity arterial disease in patients with type 2 diabetes.

\section{Methods}

\section{Patients}

Subjects were recruited from the Department of Endocrinology at Xinhua Hospital Affiliated to Shanghai Jiaotong University between 2013 and 2014. All unrelated subjects with T2DM who attended the Diabetes Clinic at the Xinhua Hospital were recruited consecutively to participate in a prospective study to identify the risk factors predisposing to the development of diabetic complications. Each visit comprised clinical assessments and laboratory investigations to determine the control of diabetes and related cardiovascular risk factors, and the presence of diabetic complications. Diabetes was defined according to the 2008 American Diabetes Association diagnostic criteria (MM) [12]. Subjects with arteriovenous grafts/shunts,vasculitis, chronic kidney disease, cerebral infarction, coronary artery disease, malignancies, or with immunological diseases, osteoporosis and subjects receiving systemic glucocorticoids or immunosuppressants were excluded from the study. A total of 712 T2DM subjects, who attended regular visits at least twice a year, with the latest follow-up in or before October 2014, were enrolled in the study. Of these, 505 patients with lower extremity arterial stenosis and 207 patients without lower extremity arterial stenosis. Written informed consent was obtained from all the participants. The study was approved by the Institutional Review Broad of Xinhua Hospital Affiliated to Shanghai Jiaotong University School of Medicine.

\section{Biochemical measurements and clinical data collection}

Peripheral venous blood samples were collected after an overnight fast. The fasting plasma glucose(FPG) was measured with the use of the glucose oxidase method on an auto-analyzer (Modular P800; Roche, Basel, Switzerland). Triglycerides, total cholesterol, LDL-cholesterol, HDLcholesterol, and serum uric acid were measured using chemiluminescence methods on the auto-analyzer (Modular E170; Roche). HbA1c was measured with the use of the Chromatography method on an auto-analyzer (D10; Bio-Rad, USA). Age and history of cigarette use were assessed through an interview preceding the physical examination. We defined "smoking" as the current smokers, who smoke at least one cigarette per day.

Anthropometric parameters were measured in all subjects. Hypertension was defined by systolic blood pressure $(\mathrm{SBP}) \geq 140 \mathrm{mmHg}$, diastolic blood pressure $(\mathrm{DBP}) \geq$ $90 \mathrm{mmHg}$, the current use of antihypertensive treatment, or a combination of the three. Three blood pressure recordings were obtained from the right arm of patients in a sitting position after $30 \mathrm{~min}$ of rest; measurements were taken in 5-min intervals, and mean values were calculated. Body mass index (BMI) was calculated as body weight $\times$ height $^{-2}$ and expressed in $\mathrm{kg} / \mathrm{m}^{2}$. The homeostasis model assessment of insulin resistance (HOMA-IR) was calculated according to the equation described by Matthews et al. [13] The waist circumference (WC) was measured at the smallest circumference between the rib cage and the iliac crest, with the subject in the standing position.

\section{Measurement of Osteoprotegerin, CRP and Adiponectin}

Plasma samples were isolated from fasting subjects and stored at $-80{ }^{\circ} \mathrm{C}$ prior to analysis. The plasma OPG, Creactive protein (CRP) and adiponectin were determined in duplicate by ELISA with Duoset kit (DY805, DY1707, and DY1065; R\&D Systems, Minneapolis, MN) as recommended by the manufacturer. The ELISA system had an intra-assay coefficient of variation of 3-9 \% and an interassay coefficient of variation of 4-10\%, respectively.

\section{Determination of lower extremity arterial disease}

The lower extremity arterial stenosis was characterized using high-frequency color Doppler ultrasonic evaluation (Philips, iU22, USA). Based on the criteria established by the 2006 American Society of Echocardiography and the Society of Vascular Medicine and Biology [14], the degree of peripheral arterial diameter reduction was classified as "normal" $(0 \%$ diameter reduction of lower extremity arterial stenosis, "mild" (1 -19\%), "moderate" (20-49\%), or "severe" (50-99\%). Current and past medical history, personal background, and medication were recorded for all subjects.

\section{Statistical analysis}

The distribution of data was tested by the KolmogorovSmirnov test. Normally distributed data were expressed as means $\pm \mathrm{SD}$, whereas variables with a skewed distribution were reported as median (inter-quartile range) and $\log$ transformed to approximate normality before analysis. Differences between groups were tested using the unpaired Student's $t$ test or $\chi^{2}$ analysis, as appropriate. 
Pearson correlations were computed to assess the relationship between variables and OPG. Plasma OPG levels were depicted according to the severity of lower extremity arterial disease using linear regression model. To investigate the association of the lower extremity arterial disease with OPG, we defined participants with normal lower extremity artery as $0(n=207)$ and the lower extremity arterial disease as $1(n=505)$ in the logistic regression analyses. Potential confounding variables including age, gender and current smoking, BMI, waist/hip ratio and HOMA-IR, SBP, DBP, CRP, HbA1c, Triglycerides, total cholesterol, HDL-C and LDL-C were controlled in the regression models. Data management and statistical analysis were performed with the SPSS Statistical Package (version 13.0; SPSS Inc., Chicago, IL). $P$ values $<0.05$ was considered statistically significant.

\section{Results}

\section{Baseline characteristics}

There were 505 (70.9 \%) patients with lower extremity arterial stenosis and 207 (29.1\%) patients without lower extremity arterial stenosis. Clinical, biochemical and metabolic characteristics of participants of the study were given in Table 1.

The plasma OPG levels were significantly increased in patients with lower extremity arterial stenosis [1.89 (1.48-2.41) vs. $2.39(1.82-3.33) \mathrm{ng} / \mathrm{mL}, p<0.001]$. Plasma OPG levels correlated positively with age $(\mathrm{r}=$ $0.410, p<0.001)$, duration of diabetes $(\mathrm{r}=0.307, p<$ $0.001), \operatorname{SBP}(\mathrm{r}=0.207, p<0.001)$ and $\mathrm{CRP}(\mathrm{r}=0.247$, $p<0.001)$. Plasma OPG levels were not associated with BMI, WC, DBP, FPG, HbA1C, uric acid, total cholesterol, triglycerides, LDL-C, HDL-C and adiponectin (Table 2).

Table 1 Characteristics of study population with or without lower extremity arterial stenosis

\begin{tabular}{|c|c|c|c|}
\hline & Without lower extremity arterial stenosis & Lower extremity arterial stenosis & $p$ value \\
\hline $\mathrm{n}$ & 207 & 505 & - \\
\hline Age (years) & $53.5 \pm 12.1$ & $65.3 \pm 11.0$ & $<0.001$ \\
\hline Gender (male/female) & $308 / 197$ & $112 / 95$ & 0.094 \\
\hline Duration of diabetes (years) & $6.3 \pm 5.9$ & $10.6 \pm 8.1$ & $<0.001$ \\
\hline smoking (\%) & $50(24.3 \%)$ & $145(28.7 \%)$ & 0.230 \\
\hline Hypertension (\%) & $86(41.5 \%)$ & $326(64.6 \%)$ & $<0.001$ \\
\hline BMI (kg/m2) & $25.1 \pm 4.2$ & $24.6 \pm 3.4$ & 0.184 \\
\hline WC (cm) & $94.2 \pm 13.1$ & $91.9 \pm 11.3$ & 0.106 \\
\hline SBP $(\mathrm{mmHg})$ & $130.2 \pm 15.1$ & $132.4 \pm 15.4$ & 0.199 \\
\hline $\mathrm{DBP}(\mathrm{mmHg})$ & $78.6 \pm 11.5$ & $76.2 \pm 9.5$ & 0.038 \\
\hline FPG $(\mathrm{mmol} / \mathrm{l})$ & $8.5 \pm 3.3$ & $8.6 \pm 3.2$ & 0.719 \\
\hline HbA1c (\%) & $9.7 \pm 2.5$ & $9.5 \pm 2.3$ & 0.490 \\
\hline$(\mathrm{mmol} / \mathrm{mol})$ & $83 \pm 19.8$ & $80 \pm 18.2$ & 0.490 \\
\hline Serum uric acid (mmol/l) & $300.3 \pm 90.7$ & $303.9 \pm 89.0$ & 0.724 \\
\hline Total cholesterol (mmol/l) & $4.5 \pm 1.1$ & $4.7 \pm 1.1$ & 0.091 \\
\hline triglycerides (mmol/l) & $1.54(1.04-2.23)$ & $1.55(1.03-2.25)$ & 0.962 \\
\hline LDL-c (mmol/l) & $2.7 \pm 0.8$ & $2.9 \pm 0.7$ & 0.122 \\
\hline $\mathrm{HDL}-\mathrm{c}(\mathrm{mmol} / \mathrm{l})$ & $1.4 \pm 0.3$ & $1.3 \pm 0.3$ & 0.203 \\
\hline Adiponectin (mg/l) & $9.2 \pm 5.7$ & $6.7 \pm 4.4$ & $<0.001$ \\
\hline CRP (mg/l) & $1.75(0.77-5.38)$ & $2.43(0.81-5.47)$ & $<0.001$ \\
\hline Osteoprotegerin (ng/ml) & $1.89(1.48-2.41)$ & $2.39(1.82-3.33)$ & $<0.001$ \\
\hline Hypoglycemic treatments & & & 0.001 \\
\hline Insulin (\%) & 78 & 188 & \\
\hline $\mathrm{OHA}(\%)$ & 100 & 186 & \\
\hline Insulin + OHA (\%) & 29 & 131 & \\
\hline
\end{tabular}

Data are means $\pm \mathrm{SD}$, medians (interquartile range), or $\mathrm{n}(\%)$. $\mathrm{P}$ values were obtained by an unpaired Student $t$ test or $X 2$ analysis, as appropriate. $B M I$ body mass index, WC waist circumference, SBP systolic blood pressure, DBP diastolic blood pressure, FPG fasting plasma glucose, $L D L-c$ low-density lipoprotein cholesterol, $H D L-c$ high-density lipoprotein cholesterol, $\mathrm{OHA}$ oral hypoglycemic agent 
Table 2 Correlation between plasma osteoprotegerin (OPG) levels and biochemical parameters

\begin{tabular}{lll}
\hline & $r$ & $p$ value \\
\hline Age & 0.410 & $<0.001$ \\
Duration of diabetes & 0.307 & $<0.001$ \\
BMI & 0.024 & 0.629 \\
WC & 0.030 & 0.553 \\
SBP & 0.207 & $<0.001$ \\
DBP & 0.015 & 0.723 \\
FPG & 0.111 & 0.011 \\
HbA1c (\%) & 0.020 & 0.680 \\
Serum uric acid & 0.005 & 0.906 \\
Total cholesterol & 0.03 & 0.521 \\
triglycerides & 0.003 & 0.953 \\
LDL-C & 0.036 & 0.423 \\
HDL-C & 0.012 & 0.792 \\
Adiponectin & -0.03 & 0.518 \\
CRP & 0.247 & $<0.001$ \\
\hline BMI body mass index WC wast
\end{tabular}

$B M I$ body mass index, $W C$ waist circumference, $S B P$ systolic blood pressure, $D B P$ diastolic blood pressure, FPG fasting plasma glucose, $L D L-c$ low-density lipoprotein cholesterol, $H D L-c$ high-density lipoprotein cholesterol

\section{Association between OPG and severity of lower extremity arterial stenosis}

The median (inter-quartile range) of OPG concentrations significantly increased for those with normal, mild, moderate and severe was 1.83 (1.41-2.29), 2.02 (1.47-2.59), 2.41 (1.74-3.45), and $2.74(2.13-3.65) \mathrm{ng} / \mathrm{mL}$, respectively, after adjustment for traditional cardiovascular risk factors such as age, gender, smoking, total cholesterol, HDL-C, CRP, BMI, and SBP $(P<0.001$ for trend), (Fig. 1$)$. The risk

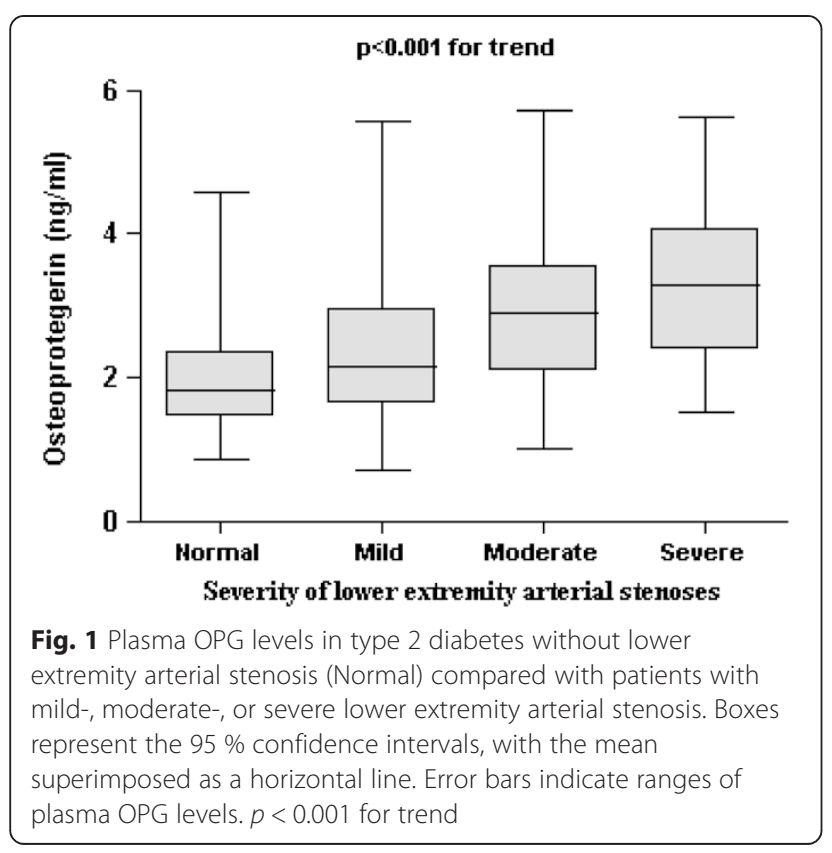

of lower extremity arterial disease was increased (OR $=1.17,95 \%$ CI 1.09-1.28, $p<0.001)$ with each SD higher level of OPG in patients with type 2 diabetes after adjustment for traditional CVD risk factors (Table 3).

\section{Discussion}

In this study, we found a strong association between plasma OPG levels and the risk of lower extremity arterial disease in patients with type 2 diabetes. Moreover, this association was independent of traditional CVD risk factors.

OPG was generally considered to be a secreted soluble receptor and was produced by many different tissues and cell types including osteoblasts. Moreover, OPG was produced by cells of the cardiovascular system, including coronary artery smooth muscle cells and endothelial cells, suggesting that alterations of plasma OPG levels may be associated with various atherosclerotic diseases, including endothelial dysfunction, arterial stiffness, aortic atheroma, carotid intima media thickness, and coronary artery disease [15-18]. In line with previous studies $[9,10]$, our findings also demonstrated that OPG had significant association with lower extremity arterial stenosis, diagnosed by high-frequency color Doppler ultrasonic, in patients with type 2 diabetes. In our study, with lower extremity arterial stenosis patients had significantly elevated plasma OPG concentrations compared with their control counterparts. Our findings, which demonstrated the association between OPG and the severity of lower extremity arterial stenosis, suggest that OPG is a potential biomarker of lower extremity arterial stenosis in type 2 diabetes. Measurement of plasma OPG could be useful for lower extremity arterial stenosis stratification of type 2 diabetes.

Table 3 The risk of lower extremity arterial stenosis associated with 1 SD increase in plasma OPG

\begin{tabular}{|c|c|c|c|}
\hline Model & Adjustment & $\begin{array}{l}\text { lower extremity } \\
\text { arterial stenosis } \\
{[\mathrm{OR},(95 \% \mathrm{Cl})]}\end{array}$ & $p$ value \\
\hline Model 1 & Unadjusted & $1.39(1.23-1.54)$ & $<0.001$ \\
\hline Model 2 & $\begin{array}{l}\text { Adjusted for age, gender } \\
\text { and current smoking }\end{array}$ & $1.35(1.18-1.49)$ & $<0.001$ \\
\hline Model 3 & $\begin{array}{l}\text { Further adjusted for BMI, } \\
\text { waist/hip ratio and } \\
\text { HOMA-IR, based on } \\
\text { Model } 2\end{array}$ & $1.24(1.11-1.40)$ & $<0.001$ \\
\hline Model 4 & $\begin{array}{l}\text { Further adjusted for } \\
\text { systolic pressure, } \\
\text { diastolic pressure, CRP, } \\
\text { HbA1c, serum } \\
\text { triglycerides, total } \\
\text { cholesterol, HDL- and } \\
\text { LDL-cholesterol, based } \\
\text { on Model } 3\end{array}$ & $1.17(1.09-1.28)$ & $<0.001$ \\
\hline
\end{tabular}


The clear mechanism for the vascular effects of OPG is unknown, however, emerging evidence indicates OPG may act as a protective factor for vascular diseases. One hypothesis is that increased serum OPG levels may be a compensatory self-defensive response to the progression of atherosclerosis $[8,19]$. It was well-documented that OPG exerts its function through binding and neutralizing the receptor activator for RANK/RANKL system [20]. RANKL can promote an osteogenic differentiation program in vascular smooth muscle cell and stimulate chemokine release, matrix metalloproteinases (MMP)-9 activity, and monocyte/macrophage matrix migration [21]. OPG functions as a soluble decoy receptor for receptor activator of RANK ligand (RANKL or OPG ligand), thereby OPG may serve a protective role in the vascular system. However,some studies indicated OPG may play causal role in atherosclerotic disease. Furthermore, OPG is also a receptor for the cytotoxic ligand TNF-related apoptosis inducing ligand (TRAIL), a potent activator of apoptosis. Recently, OPG has been identified as a survival factor for endothelial cells by blocking TRAIL-induced apoptosis. The regulatory mechanism of OPG on vascular calcification could promote the progression and instability of atherosclerosis [22, 23]. Moreover, a serial of clinical studies have shown that elevated OPG is associated with a higher risk for death and worse clinical outcome in patients with cardiovascular disease $[24,25]$. Furthermore, increased OPG level is an independent risk factor for further progression of carotid atherosclerosis in prospective studies [26]. Although we could not determine whether OPG has a protective or promoting role in lower extremity arterial stenosis by the limitation of design of this study, our findings suggested that the OPG might have a role as a biomarker to identifying patient with lower extremity arterial stenosis.

We found that plasma OPG levels were positively correlated with age, duration of diabetes, SBP, CRP. OPG was not associated with BMI, WC, DBP, FPG, HbA1c, uric acid, total cholesterol, triglycerides, LDL-C, HDL-C and adiponectin. The positive relation we found between OPG and age confirmed the results of previous studies in both men and women [27]. This finding suggested that the factors associated with aging may regulate OPG levels. Szulc et al. showed that the relation between OPG and age was more relevant in men by age 40-45 years, at a time in life when age-dependent bone loss begined [28]. The increasing serum OPG level with ageing could be interpreted as a compensatory mechanism counteracting the age progression of bone resorption and atherosclerosis.

To our knowledge, our study is the first one to look at a relationship between OPG and the severity of lower extremity arterial stenosis. However, this study had a number of limitations. First, a prospective study is needed to clarify the causal relationship between OPG and lower extremity arterial stenosis. Second, the relatively small sample size limits the generalizability of our conclusions. Further studies looking at these relations in larger populations such as old and young people should be performed. Third is the fact that circulating concentrations of biomarkers are influenced by a number of factors, mostly unknown, and their usage should be validated in each condition.

\section{Conclusions}

In summary, our data showed that, increased plasma OPG levels were independently associated with the presence and the severity of lower extremity arterial stenosis. This finding suggested that plasma OPG levels were an important determinant of lower extremity arterial atherosclerosis in patients with type 2 diabetes. Although the exact pathophysiologic effect of OPG on lower extremity arterial stenosis is unknown, our findings suggest that plasma OPG is a potential biomarker for lower extremity arterial stenosis. Further studies are warranted to determine the functional role of OPG in the development of atherosclerosis in normal patients.

\section{Competing interests}

The authors declare that they have no competing interests.

\section{Authors' contributions}

Conceived and designed the experiments: QS. Analyzed the data: YN, WZ, $Z Y$. Contributed reagents/materials/analysis tools: YN, WZ, ZY, XL, JW, HZ, SW, $X W, H Z, W F, L Q$. Wrote the paper: YN, ZY. All authors read and approved the final manuscript.

\section{Acknowledgements}

This work was supported by the Shanghai Science and Technology Commission (10411956600, 14ZR1427400), Natural Science Foundation of Shanghai (11ZR1405300), National Natural Science Foundation of China (81300667, 81000332, 81170322, 81370953, 81370935), Shanghai Health System Outstanding Young Talents Training Program (XYQ2013098), Chinesse Society of Endocrinology.

\section{Author details}

${ }^{1}$ Department of Endocrinology, Xinhua Hospital Affiliated to Shanghai Jiaotong University School of Medicine, 1665 Kongjiang Road, Shanghai, China. ${ }^{2}$ Department of Endocrinology, Huashan Hospital Affiliated to Fudan University, Shanghai, China. ${ }^{3}$ Department of Endocrinology, Clinical Geriatric Medicine, Henan Provincial People's Hospital, Zhengzhou, China.

${ }^{4}$ Department of Geriatrics, Huashan Hospital Affiliated to Fudan University, Shanghai, China.

Received: 21 April 2015 Accepted: 29 July 2015

Published online: 11 August 2015

\section{References}

1. Rasmussen LM, Ledet T. Osteoprotegerin and diabetic macroangiopathy. Horm Metab Res. 2005;37:90-4.

2. Jono S, Ikari Y, Shioi A, Mori K, Miki T, Hara K, et al. Serum osteoprotegerin levels are associated with the presence and severity of coronary artery disease. Circulation. 2002;106:1192-4.

3. Kim J, Song TJ, Yang SH, Lee OH, Nam HS, Kim YD, et al. Plasma osteoprotegerin levels increase with the severity of cerebral artery atherosclerosis. Clin Biochem. 2013;46:1036-40.

4. Lieb W, Gona P, Larson MG, Massaro JM, Lipinska I, Keaney Jr JF, et al. Biomarkers of the osteoprotegerin pathway: clinical correlates, subclinical 
disease, incident cardiovascular disease, and mortality. Arterioscler Thromb Vasc Biol. 2010;30:1849-54.

5. Stepien E, Wypasek E, Stopyra K, Konieczyńska M, Przybyło M, Pasowicz M. Increased levels of bone remodeling biomarkers (osteoprotegerin and osteopontin)in hypertensive individuals. Clin Biochem. 2011;44:826-31.

6. Bucay N, Sarosi I, Dunstan CR, Morony S, Tarpley J, Capparelli C, et al. Osteoprotegerin-deficient mice develop early onset osteoporosis and arterial calcification. Genes Dev. 1998;12:1260-8.

7. Kim SM, Lee J, Ryu OH, Lee KW, Kim HY, Seo JA, et al. Serum osteoprotegerin levels are associated with inflammation and pulse wave velocity. Clin Endocrinol. 2005;63:594-8.

8. Schoppet M, Sattler AM, Schaefer JR, Herzum M, Maisch B, Hofbauer LC. Increased osteoprotegerin serum levels in men with coronary artery disease. J Clin Endocrinol Metab. 2003;88:1024-8.

9. Poulsen MK, Nybo M, Dahl J, Hosbond S, Poulsen TS, Johansen A, et al. Plasma osteoprotegerin is related to carotid and peripheral arterial disease, but not to myocardial ischemia in type 2 diabetes mellitus. Cardiovasc Diabetol. 2011;10:76.

10. O'Sullivan EP, Ashley DT, Davenport C, Kelly J, Devlin N, Crowley R, et al. Osteoprotegerin is higher in peripheral arterial disease regardless of glycaemic status. Thromb Res. 2010;126:e423-427.

11. Hosbond SE, Poulsen TS, Diederichsen AC, Nybo M, Rasmussen LM, Mickley H. Osteoprotegerin as a marker of atherosclerosis: a systematic update. Scand Cardiovasc J. 2012;46:203-11.

12. Report of the Expert Committee on the Diagnosis and Classification of Diabetes Mellitus. (1997). Diabetes Care, 20, 1183-1197.

13. Matthews DR, Hosker JP, Rudenski AS, Naylor BA, Treacher DF, Turner RC Homeostasis model assessment: insulin resistance and beta-cell function from fasting plasma glucose and insulin concentrations in man. Diabetologia. 1985;28:412-9.

14. Gerhard-Herman M, Gardin JM, Jaff M, Mohler E, Roman M, Naqvi TZ. Guidelines for noninvasive vascular laboratory testing: A report from the American Society of Echocardiography and the Society of Vascular Medicine and Biology. J Am Soc Echocardiogr. 2006;19:955-72.

15. Kiechl S, Werner $P$, Knoflach M, Furtner M, Willeit J, Schett G. The osteoprotegerin /RANK/RANKL system: a bone key to vascular disease. Expert Rev Cardiovasc Ther. 2006;:801-11.

16. Ali Z, Ellington AA, Mosley Jr TH, Kullo IJ. Association of serum osteoprotegerin with ankle-brachial index and urine albumin: creatinine ratio in African-Americans and non-Hispanic whites. Atherosclerosis. 2009;206:575-80

17. Vik A, Mathiesen EB, Brox J, Wilsgaard T, Njølstad I, Jørgensen L, et al. Relation between serum osteoprotegerin and carotid intima media thickness in a general population - the Tromsø Study. J Thromb Haemost. 2010;8:2133-9.

18. Gordin D, Soro-Paavonen A, Thomas MC, Harjutsalo V, Saraheimo M, Bjerre M, Forsblom C, Flyvbjerg A, Groop PH, FinnDiane Study Group. Osteoprotegerin is an independent predictor of vascular events in Finnish adults with type 1 diabetes. Diabetes Care. 2013;36:1827-33.

19. Schoppet M, Preissner KT, Hofbauer LC. RANK ligand and osteoprotegerin. Paracrine regulators of bone metabolism and vascular function. Arterioscler Thromb Vasc Biol. 2002;22:549-53.

20. Zhou S, Fang X, Xin H, Li W, Qiu H, Guan S. Osteoprotegerin inhibits calcification of vascular smooth muscle cell via down regulation of the Notch1-RBP-JK/MsX2 signaling pathway. PLoS ONE. 2013;8, e68987.

21. Collin-Osdoby P. Regulation of vascular calcification by osteoclast regulatory factors rankl and osteoprotegerin. Circ Res. 2004;95:1046-57.

22. Tousoulis D, Siasos G, Maniatis K, Oikonomou E, Kioufis S, Zaromitidou M, et al. Serum osteoprotegerin and osteopontin levels are associated with arterial stiffness and the presence and severity of coronary artery disease. Int J Cardiol. 2013;167:1924-8.

23. Sattler AM, Schoppet M, Schaefer JR, Hofbauer LC. Novel aspects on RANK ligand and osteoprotegerin in osteoporosis and vascular disease. Calcif Tissue Int. 2004;74:103-6

24. Rogers A, Eastell R. REVIEW: Circulating osteoprotegerin and receptor activator for nuclear factor-KB ligand: clinical utility in metabolic bone disease assessment. J Clin Endocrinol Metab. 2005;90:6323-31.

25. Kadoglou NP, Papadakis I, Moulakakis KG, Ikonomidis I, Alepaki M, Moustardas $\mathrm{P}$, et al. Arterial stiffness and novel biomarkers in patients with abdominal aortic aneurysms. Regul Pept. 2012;179:50-4.
26. Kiechl S, Schett G, Wenning G, Redlich K, Oberhollenzer M, Mayr A, et al. Osteoprotegerinis a risk factor for progressive atherosclerosis and cardiovascular disease. Circulation. 2004;109:2175-80.

27. Gannagé-Yared MH, Fares F, Semaan M, Khalife S, Jambart S. Circulating osteoprotegerin is correlated with lipid profile, insulin sensitivity, adiponectin and sex steroids in an ageing male population. Clin Endocrinol. 2006;64:652-8

28. Szulc P, Hofbauer LC, Heufelder AE, Roth S, Delmas PD. Osteoprotegerin serum in men: correlation with age, Estrogen, and testosterone status. J Clin Endocrinol Metab. 2001;86:3162-5.

\section{Submit your next manuscript to BioMed Central and take full advantage of:}

- Convenient online submission

- Thorough peer review

- No space constraints or color figure charges

- Immediate publication on acceptance

- Inclusion in PubMed, CAS, Scopus and Google Scholar

- Research which is freely available for redistribution

Submit your manuscript at www.biomedcentral.com/submit 